\title{
A!
}

This is an electronic reprint of the original article.

This reprint may differ from the original in pagination and typographic detail.

Havu, P.; Tuomisto, N.; Väänänen, R.; Puska, M.J.; Nieminen, R.M.

\section{Spin dependent electron transport through a magnetic resonant tunneling diode}

Published in:

Physical Review B

DOI:

10.1103/PhysRevB.71.235301

Published: 02/06/2005

Document Version

Publisher's PDF, also known as Version of record

Published under the following license:

Unspecified

Please cite the original version:

Havu, P., Tuomisto, N., Väänänen, R., Puska, M. J., \& Nieminen, R. M. (2005). Spin dependent electron transport through a magnetic resonant tunneling diode. Physical Review B, 71(23), 1-11. [235301].

https://doi.org/10.1103/PhysRevB.71.235301

This material is protected by copyright and other intellectual property rights, and duplication or sale of all or part of any of the repository collections is not permitted, except that material may be duplicated by you for your research use or educational purposes in electronic or print form. You must obtain permission for any other use. Electronic or print copies may not be offered, whether for sale or otherwise to anyone who is not an authorised user. 


\title{
Spin-dependent electron transport through a magnetic resonant tunneling diode
}

\author{
P. Havu, N. Tuomisto, R. Väänänen, M. J. Puska, and R. M. Nieminen \\ Laboratory of Physics, Helsinki University of Technology, P.O. Box 1100, FIN-02015 HUT, Finland
}

(Received 23 November 2004; revised manuscript received 11 February 2005; published 2 June 2005)

\begin{abstract}
Electron-transport properties in nanostructures can be modeled, for example, by using the semiclassical Wigner formalism or the quantum-mechanical Green's function formalism. We compare the performance and the results of these methods in the case of magnetic resonant-tunneling diodes. We have implemented the two methods within the self-consistent spin-density-functional theory. Our numerical implementation of the Wigner formalism is based on the finite-difference scheme whereas for the Green's function formalism the finiteelement method is used. As a specific application, we consider the device studied by Slobodskyy et al. [Phys. Rev. Lett. 90, 246601 (2003)] and analyze their experimental results. The Wigner and Green's function formalisms give similar electron densities and potentials but, surprisingly, the former method requires much more computer resources in order to obtain numerically accurate results for currents. Both of the formalisms can be used to model magnetic resonant tunneling diode structures.
\end{abstract}

DOI: $10.1103 /$ PhysRevB.71.235301

PACS number(s): 73.63.-b, 75.47.-m

\section{INTRODUCTION}

The future spintronics technology requires controlled spin injection into semiconductor materials. The problem can be solved using different techniques and constructions (see, for example, Refs. 1-5). The magnetic resonant tunneling diode (RTD) suggested by Egues on the basis of model calculations ${ }^{23}$ and demonstrated experimentally by Slobodskyy et al. ${ }^{6}$ is one of the most promising solutions. The magnetic RTD is based on the quantum well made of dilute magnetic semiconductor $\mathrm{ZnMnSe}$ between two ZnBeSe barriers and surrounded by highly $n$-type ZnSe. In the presence of a magnetic field there is a giant Zeeman splitting between the spin-up and spin-down electron states in the quantum well region. The corresponding resonance peaks in the electron current separate as a function of the bias voltage, and the device controls the electron spin states using the bias voltage rather than external magnetic fields. This is a useful property for possible practical applications.

In a typical nanoscale transport problem two or more electrodes are connected to a functional nanostructure. An important nanosystem is the RTD composed of layers of semiconducting materials. The offsets between the band edges of the materials give rise to the two potential barriers seen by carriers. In the quantum well between the barriers, resonance states with finite energy width and enhanced amplitude are formed. The electron current increases rapidly when a resonance state appears in the conducting window, determined by the overlap of the occupied source and unoccupied drain conduction electron bands. When the resonance state drops with increasing bias voltage below the source conduction band, the current diminishes causing a region of negative resistance. In addition to being technologically interesting the RTD is also important as a simple test case for different formalisms and computational schemes.

Theoretical modeling and computational simulation are essential for the development of functional nanostructures. Electron transport properties can be modeled using different formalisms at different levels of sophistication. Two methods widely used are the Wigner function (WF ${ }^{7,9}$ and the Green's function (GF) formalism. ${ }^{9}$ The WF approach is a semiclassical transport formalism that enables the study of systems exhibiting quantum interference and tunneling effects. The electron density and the current are obtained from the Wigner function, which is in turn calculated by solving the Liouville-von Neumann equation. The GF formalism is a fully quantum-mechanical scheme with an increased complexity with respect to the WF formalism. Both the WF and GF formalisms enable self-consistent nonequilibrium calculations corresponding to a finite bias voltage between the electrodes.

RTD's can be modeled as structures which are translationally invariant parallel to the layers. This makes the computations one-dimensional. The WF and GF formalisms are very popular schemes in their modeling (see, for example, Refs. 10-13). In this work we study the feasibility of the WF and GF formalisms within the context of the self-consistent spin-density-functional theory (SDFT) and its local spindensity approximation to model magnetic RTD's. Our implementation of the WF formalism is based on the usual discretation of the partial differential equations on position and momentum point grids. Our implementation of the GF formalism employs the finite-element method (FEM). We have already published our FEM scheme for two-dimensional nanostructures ${ }^{14}$ and used it in applications. ${ }^{15}$ In this work we critically compare the performance and the results of the WF and GF implementations for quasi-one-dimensional RTDs. Moreover, we choose the structure parameters of our test system to correspond the magnetic RTD device by Slobodskyy et al. ${ }^{6}$ Thus, we can compare our results also with experiments and actually analyze the results of the measurements.

Below we use the effective atomic units which are derived by setting the fundamental constants $e=\hbar=m_{e}=1$, and the material constants $m^{*}=\epsilon=1 . m^{*}$ and $\epsilon$ are, respectively, the relative effective electron mass and the relative dielectric constant to be used in the effective mass approximation. For ZnSe $m^{*}=0.145$ and $\epsilon=9.1{ }^{16,17}$ We have used same values also for the other materials of the magnetic RTD. The effec- 


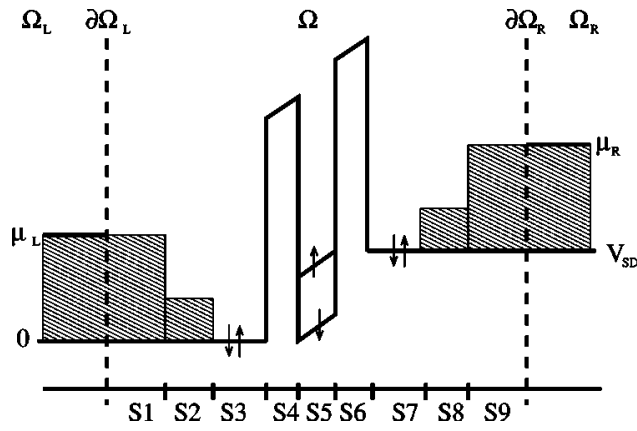

FIG. 1. Magnetic RTD model. The shaded areas denote positive background charge. The detailed information about the different layers $\mathrm{S} 1, \ldots, \mathrm{S} 9$ are given in Table I.

tive atomic units can be transformed to the usual atomic and SI units using the relations

$$
\begin{gathered}
\text { Length: } 1 a_{0}^{*}=1 \frac{\epsilon}{m^{*}} a_{0} \approx 3.32 \mathrm{~nm}, \\
\text { Energy: } 1 \mathrm{Ha}^{*}=1 \frac{m^{*}}{\epsilon^{2}} \mathrm{Ha} \approx 47.6 \mathrm{meV}, \\
\text { Current: } 1 \text { a.u. } *=1 \frac{m^{*}}{\epsilon^{2}} \text { a.u. } \approx 11.6 \mu \mathrm{A} .
\end{gathered}
$$

Above, Ha denotes the Hartree energy unit.

In Sec. II we introduce the model for the magnetic RTD and explain the use of the SDFT in the calculations. In Sec. III we briefly explain the GF and WF formalisms. In Sec. IV we give the results of the comparisons between the two formalisms and compare the calculated and measured results and obtain information on the electronic structure of the device in question. Section $\mathrm{V}$ contains our conclusions.

\section{MODEL}

\section{A. Structure of magnetic RTD}

The model for the magnetic RTD is shown in Fig. 1. We assume the semiconducting layers to be infinitely wide in the lateral directions so that the system is translationally invariant in the direction perpendicular to the current. The doped regions (shadowed areas in Fig. 1) are modeled by a uniform positive background charge. The potential barriers due to the discontinuity of the conduction band between two materials are described by constant external potentials.

The system is divided into the central region $\Omega$ and the outside regions $\Omega_{L}$ and $\Omega_{R}$. All the structural variations and interesting phenomena take place in region $\Omega$ which is chosen large enough so that the effect of the RTD device on the electron density has vanished at the boundaries $\partial \Omega_{L / R} . \Omega_{L / R}$ are the semi-infinite leads where the electron density and the potential are constant. The bottom of the conduction band and the Fermi-level $\mu_{R}$ in the right lead are shifted by the bias voltage $V_{S D}$ with respect to the corresponding values in the left lead (see Fig. 1). In our model the electron transport
TABLE I. Parameters of the different layers $\mathrm{S} 1, \ldots, \mathrm{S} 9$ used in the calculations.

\begin{tabular}{ccccc}
\hline \hline Region & Material & $\begin{array}{c}\text { Width } \\
(\mathrm{nm})\end{array}$ & $\begin{array}{c}\text { Doping level } \\
\left(\mathrm{cm}^{-3}\right)\end{array}$ & $\begin{array}{c}\text { Potential } \\
V_{W}(\mathrm{meV})\end{array}$ \\
\hline S1, S9 & $\mathrm{ZnSe}$ & 25 & $15 \times 10^{18}$ & 0 \\
S2, S8 & $\mathrm{Zn}_{0.97} \mathrm{Be}_{0.03} \mathrm{Se}$ & 15 & $1 \times 10^{18}$ & 0 \\
S3, S7 & $\left.\mathrm{ZnSe}^{18}\right)$ & 10 & 0 & 0 \\
S4, S6 & $\mathrm{Zn}_{0.7} \mathrm{Be}_{0.3} \mathrm{Se}$ & 5 & 0 & 92 \\
S5 & $\mathrm{Zn}_{0.96} \mathrm{Mn}_{0.08} \mathrm{Se}$ & 9 & 0 & $\pm \frac{1}{2} \Delta E$ \\
\hline \hline
\end{tabular}

is ballistic with no phonon or defect scattering. This means that the total potential drop takes place within $\Omega$.

In addition, $\Omega$ is divided into nine smaller parts $S 1, \ldots, S 9$ as shown in Fig. 1. These regions describe different semiconductor material layers. The parameters of the layers are given in Table I. We have chosen the widths and the doping densities of our magnetic RTD structure similar to those in the actual device made by Slobodskyy et al. ${ }^{6}$ The positive background charge densities in regions $\mathrm{S} 1$ and $\mathrm{S} 9$ are equal to that in $\Omega_{L / R}$, in $\mathrm{S} 2$ and $\mathrm{S} 8$ they are much smaller, and next to the potential barriers there is no background charge at all.

The regions S4 and S6 are the potential barriers. Because there is no definitive information about the barrier height we estimate it by comparing the shapes of the calculated currentvoltage curves to the measured ones. By the shape we mean mainly the widths of the resonances; the positions of the resonances are quite insensitive to the barrier height. The barrier height is the only structural parameter which we have to determine by fitting. We find that the barrier height of $23 \%$ of the band gap difference ${ }^{18,19}$ between $\mathrm{ZnSe}$ and $\mathrm{ZnBeSe}$ results in a good fit. Moreover, in order to estimate effects of the impurity band formation in the highly doped regions we have in some test calculations tried to shift the bottom of the bands (and Fermi levels) in the highly doped regions with respect to those in the insulating ones.

The quantum well S5 is made of the dilute magnetic semiconductor ZnMnSe. An external magnetic field causes a giant Zeeman effect, splitting the spin-up and spin-down electron states. In the other parts of the RTD the spin splitting is small and assumed to vanish. We calculate the spin splitting $\Delta E$ in S5 as Slobodskyy et al., ${ }^{6}$ i.e.,

$$
\Delta E=N_{0} \alpha x s_{0} B_{s}\left(\frac{s g \mu_{B} B}{k_{B}\left(T+T_{\mathrm{eff}}\right)}\right) .
$$

Above, $N_{0} \alpha$ is the $s$ - $d$ exchange integral, $x$ the Mn concentration, $g$ is the Landé factor, $\mu_{B}$ the Bohr magneton, $B_{s}$ is the Brillouin function of spin $s, s_{0}$ is the effective Mn spin, and $T_{\text {eff }}$ is the effective temperature. The values of the parameters are $N_{0} \alpha=0.26 \mathrm{eV}, \quad x=8 \%, s=5 / 2, g=2.00$, $s_{0}=1.13$, and $T_{\text {eff }}=2.24 \mathrm{~K}$. The values of the $\Delta E$ for the relevant magnetic fields and temperatures (Sec. V) are collected into Table II. 
TABLE II. Values of the spin splitting $\Delta E$ in the cases considered in Sec. V.

\begin{tabular}{ccc}
\hline \hline Magnetic field $(\mathrm{T})$ & Temperature $(\mathrm{K})$ & $\Delta E(\mathrm{meV})$ \\
\hline 0 & 4.2 & 0.0 \\
2 & 4.2 & 10.4 \\
4 & 4.2 & 16.7 \\
6 & 4.2 & 19.8 \\
6 & 0 & 23.2 \\
6 & 8 & 16.2 \\
6 & 30 & 6.7 \\
\hline \hline
\end{tabular}

\section{B. Spin-density-functional theory}

In order to model electron-electron interactions we use the SDFT within the local-density approximation. The electronic structures and currents are calculated using the GF or the WF formalisms, which we will explain later in Sec. III. In both formalisms the spin-up $(\sigma=\uparrow)$ and spin-down $(\sigma=\downarrow)$ electron densities $n_{\uparrow, \downarrow}(x)$ correspond to the effective potentials

$$
V_{\mathrm{eff}}^{\sigma}(x)=V_{C}(x)+V_{\mathrm{xc}}^{\sigma}(x)+V_{W}^{\sigma}(x),
$$

where $x$ is the coordinate perpendicular to the layers, $V_{C}$ is the Coulomb potential, $V_{\mathrm{xc}}^{\sigma}$ the exchange-correlation potential, ${ }^{20}$ and $V_{W}^{\sigma}$ the external potential including the barriers and the giant Zeeman spin splitting $\Delta E$ (see Table II). The spin densities and the effective potentials are solved self-consistently.

The Coulomb potential $V_{C}$ is calculated using the modified Poisson equation ${ }^{21}$

$$
\nabla^{2} V_{C}^{i+1}(x)-k^{2} V_{C}^{i+1}(x)=-4 \pi\left[n+(x)-n_{-}^{i}(x)\right]-k^{2} V_{C}^{i}(x),
$$

where $n_{-}(x)=n_{\uparrow}(x)+n_{\downarrow}(x)$ is the total electron density and $n_{+}(x)$ is the positive background charge. Index $i$ counts the self-consistency iterations, so that $V_{C}^{i}(x)$ is the solution from the previous self-consistency iteration. Above, $k$ is a parameter which controls the screening of the potential fluctuations due to the charge sloshing between the iterations. A reasonable choice of the $k$ parameter is of the order of the Thomas-Fermi wave vector, in which case the solution does not depend on $k$ and the number of the self-consistency iterations needed is remarkably reduced. In addition, the stability obtained by the use of the modified Poisson equation, we stabilize the iterations also by mixing the old effective potential $V_{\text {eff }}^{i}$ with that obtained from Eqs. (2) and (3). That is,

$$
V_{\mathrm{eff}}^{i+1}=\alpha V_{\mathrm{eff}}+(1-\alpha) V_{\mathrm{eff}}^{i},
$$

where the feedback parameter $\alpha$ is typically 0.2 in our calculations.

We calculate the currents due to the spin-up and spindown electrons through the magnetic RTD as a function of the voltage. The calculation always starts from the zero bias voltage $V_{S D}$. When the self-consistent nonbiased (equilib- rium) result is reached, we increase $V_{S D}$ in small steps and iterate at every value until convergence. The effective potential corresponding to the previous $V_{S D}$ value is used as the starting point of the iterations. This ensures the stability of the process. Actually we found that the resulting current voltage curve does not depend on the sweep direction of the bias voltage. This is due to the very small electron density in the quantum well region S5 even in the case of occupied resonance states. This insensitivity is in accord with the findings by Slobodskyy et al. ${ }^{6}$

\section{FORMALISMS}

In this section we present the GF and WF formalisms by ignoring the spin dependence for simplicity. The generalization to the spin-dependent forms, which we use in the actual calculations, is straightforward.

\section{A. Green's function formalism}

The GF formalism used in the electron density and transport calculations is explained in detail in Ref. 9. We have implemented this formalism using the finite-element method (FEM). Our FEM formulation for two-dimensional nanostructures is discussed in Ref. 14.

Our present magnetic RTD system is translationally invariant in directions perpendicular to the electron current. The total electron energy $\omega_{\text {tot }}$ can then be divided into two parts

$$
\omega_{\text {tot }}=\omega+\omega_{\perp}
$$

where $\omega_{\perp}$ is the kinetic energy in the perpendicular directions and $\omega$ includes the kinetic energy along the current and the (one-dimensional) potential energy. Now we can write a one-dimensional equation for the single-particle retarded Green's function in the real space using spatial coordinates, $x$ and $x^{\prime}$ in the direction perpendicular to the layers

$$
(\omega-\hat{H}) G^{r}\left(x, x^{\prime} ; \omega\right)=\delta\left(x-x^{\prime}\right),
$$

where $\hat{H}$ is the Hamiltonian

$$
\hat{H}=-\frac{1}{2} \nabla^{2}+V_{\text {eff }}(x)
$$

Above, $\omega$ has a small imaginary part, i.e., $\omega=\omega^{\prime}+i \eta$. This distinguishes between the retarded and the advanced Green's functions. Equation (6) is solved using open boundary conditions at $\partial \Omega_{L}$ and $\partial \Omega_{R}$. This means that electron wave functions penetrate the boundaries without reflection. In order to restrict the numerical calculations into the central region $\Omega$ Eq. (6) is written in the form

$$
\left[\omega-\hat{H}_{0}-\Sigma_{L}^{r}(\omega)-\Sigma_{R}^{r}(\omega)\right] G^{r}\left(x, x^{\prime} ; \omega\right)=\delta\left(x-x^{\prime}\right),
$$

where $\Sigma_{L / R}^{r}(\omega)$ are the self-energies of the leads $\Omega_{L / R}$ and $\hat{H}_{0}$ is the Hamiltonian of the isolated region $\Omega$.

The electron density is obtained from the so-called lesser Green function $G^{<}$by integrating over $\omega$ : 


$$
n_{-}(x)=\frac{-1}{2 \pi} \int_{-\infty}^{\infty} \operatorname{Im}\left[G^{<}(x, x ; \omega)\right] d \omega .
$$

Notice that we assume the parabolic free electron states in the perpendicular directions and a constant two-dimensional density of states is used. When no bias voltage is applied the system is in equilibrium and $G^{<}$is calculated as

$$
G^{<}\left(x, x^{\prime} ; \omega\right)=2 F_{L / R}(\omega) G^{r}\left(x, x^{\prime} ; \omega\right) .
$$

Above, $F_{L / R}$ are related to the Fermi distributions in $\Omega_{L / R}$ and in the equilibrium they are equal. The effects of the perpendicular directions in our computationally one-dimensional system are included in $F_{L / R}$ by integrating the Fermi distributions over $\omega_{\perp}$

$$
\begin{aligned}
F_{L / R}(\omega) & =\frac{1}{\pi} \int_{0}^{\infty} \frac{1}{1+e^{\left(\omega+\omega_{\perp}-\mu_{L / R}\right) / k_{B} T}} d \omega_{\perp} \\
& =\frac{1}{\pi} k_{B} T \ln \left(1+e^{\mu_{L / R^{-\omega}} / k_{B} T}\right) .
\end{aligned}
$$

Notice that we assume above parabolic free electron states in the perpendicular directions and a constant two-dimensional density of states is used. When the bias voltage $V_{S D}$ is applied, $F_{L}(\omega)$ and $F_{R}(\omega)$ are split by $V_{S D}$ on the energy axis. In this case $G^{<}$has to be calculated as

$$
\begin{aligned}
G^{<}\left(x, x^{\prime} ; \omega\right)= & -i F_{R}(\omega) G^{r}\left(x, x_{R} ; \omega\right) \Gamma_{R}\left(x_{R}, x_{R}^{\prime} ; \omega\right) G^{a}\left(x_{R}^{\prime}, x^{\prime} ; \omega\right) \\
& -i F_{L}(\omega) G^{r}\left(x, x_{L} ; \omega\right) \Gamma_{L}\left(x_{L}, x_{L}^{\prime} ; \omega\right) G^{a}\left(x_{L}^{\prime}, x^{\prime} ; \omega\right),
\end{aligned}
$$

where $x_{L / R}$ are the coordinates of the boundaries $\partial \Omega_{L / R}$ and $\Gamma_{L / R}$ are defined as

$$
i \Gamma_{L / R}=\Sigma_{L / R}^{r}-\Sigma_{L / R}^{a}=2 i \operatorname{Im}\left(\Sigma_{L / R}^{r}\right) .
$$

Equation (12) is valid also in equilibrium, but only when there are no bound states. Namely, in this form the electron density in $\Omega$ is composed of scattering electron states coming from $\Omega_{L}$ and $\Omega_{R}$.

To perform the energy integral in Eq. (9) is the heaviest part of the calculations. The calculation of $G^{<}$at several energies $\omega$ takes CPU time but cannot be avoided due to the sharp resonance peaks in our RTD system. In order to reduce the number of $\omega$ values needed we move parts of the integral to the complex plane where the changes in $G^{<}$are smooth. The move of the integration part away from the real axis requires that the integrand is analytic above the real axis. To fulfill this we first approximate Eq. (11) as

$$
F_{L / R}(\omega) \approx \frac{\mu_{L / R}-\omega}{\pi} .
$$

This is exactly true at zero temperature and a good approximation at energies few $k_{B} T$ below the Fermi levels $\mu_{L / R}$. Next we write Eq. (12) in the form

$$
\begin{aligned}
G^{<}\left(x, x^{\prime}\right)= & -i F_{L}\left[G^{r}\left(x, x_{R}\right) \Gamma_{R}\left(x_{R}, x_{R}^{\prime}\right) G^{a}\left(x_{R}^{\prime}, x^{\prime}\right)\right. \\
& \left.+G^{r}\left(x, x_{L}\right) \Gamma_{L}\left(x_{L}, x_{L}^{\prime}\right) G^{a}\left(x_{L}^{\prime}, x^{\prime}\right)\right],
\end{aligned}
$$

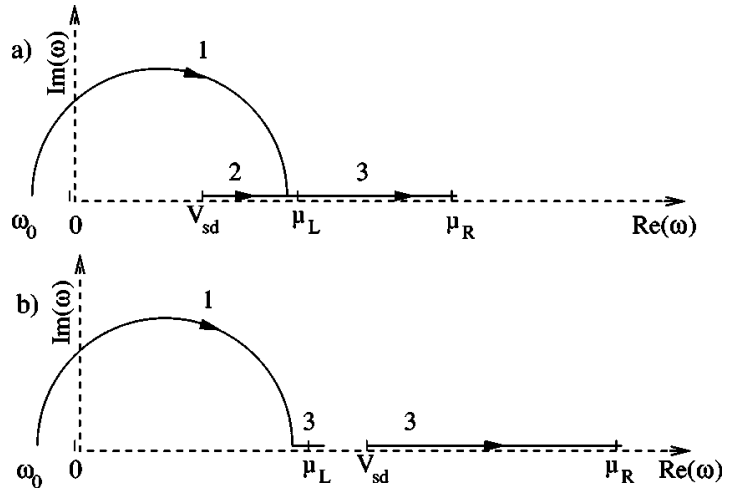

FIG. 2. Integration paths for the electron density calculations. The paths are divided into three parts $n_{-1}, n_{-2}$, and $n_{-3}$. (a) and (b) correspond to the cases $V_{S D}<\mu_{L}$ and $V_{S D}>\mu_{L}$, respectively.

$$
-i\left[F_{R}-F_{L}\right] G^{r}\left(x, x_{R}\right) \Gamma_{L}\left(x_{R}, x_{R}^{\prime}\right) G^{a}\left(x_{R}^{\prime}, x^{\prime}\right),
$$

where the $\omega$ dependences are not shown for the clarity. Now we can split the integral (9) into three parts $n_{-1}, n_{-2}$, and $n_{-3}$ shown in Fig. 2. The slightly complicated division is due to the proper inclusion of the perpendicular kinetic energy component. Here and below we assume that $\mu_{L} \leqslant \mu_{R}$. In the first part $n_{-1}$ comes from the first term in Eq. (15), where the form of $G^{<}$in Eq. (10) is used and the integral

$$
n_{-1}=\int_{\omega_{0}}^{\mu_{L^{-}-\Delta k_{B} T}} \frac{1}{\pi}\left(\mu_{L}-\omega\right) \operatorname{Im}\left[G^{r}\left(x, x^{\prime} ; \omega\right)\right] d \omega
$$

is moved to the complex plane. Above, $\Delta$ is about $3, \ldots, 5$ so that Eq. (14) is approximately valid. The integral starts at the energy $\omega_{0}$, which is below the bottom of the conduction band of the left lead so that the contribution of possible bound states is also included. The integral ends at a couple of $k_{B} T$ below the Fermi level $\mu_{L}$.

The second term in Eq. (15) yields the integral.

$$
\begin{aligned}
n_{-2}= & \int_{\omega_{0}+\mu_{R}-\mu_{L}}^{\mu_{L}-\Delta k_{B} T} \frac{1}{\pi}\left(\mu_{R}-\mu_{L}\right) \\
& \times G^{r}\left(x, x_{R} ; \omega\right) \Gamma_{R}\left(x_{R}, x_{R}^{\prime} ; \omega\right) G^{a}\left(x_{R}^{\prime}, x^{\prime} ; \omega\right) d \omega .
\end{aligned}
$$

[Note: $\Gamma_{R}(\omega)=0$ for $\omega<V_{S D}$.] Because Eq. (12) has to be used the integrand is not analytic on the complex plane and the integration has to be performed along the real axis. The integral $n_{-2}$ vanish when $V_{S D}=0$ and $V_{S D}>\mu_{L}$ [the case of Fig. 2(a)].

The integral $n_{-3}$ starts at a couple of $k_{B} T$ below $\mu_{L}$ and ends at a couple of $k_{B} T$ above $\mu_{R}$. It is obtained as

$$
\begin{aligned}
n_{-3}= & \frac{1}{2 \pi} \int_{\mu_{L}-\Delta k_{B} T}^{\mu_{R^{+} \Delta k_{B} T}}\left[F_{R}(\omega) G^{r}\left(x, x_{R} ; \omega\right) \Gamma_{R}\left(x_{R}, x_{R}^{\prime} ; \omega\right)\right. \\
& \times G^{a}\left(x_{R}^{\prime}, x^{\prime} ; \omega\right)+F_{L}(\omega) G^{r}\left(x, x_{L} ; \omega\right) \\
& \left.\times \Gamma_{L}\left(x_{L}, x_{L}^{\prime} ; \omega\right) G^{a}\left(x_{L}^{\prime}, x^{\prime} ; \omega\right)\right] d \omega .
\end{aligned}
$$

When $V_{S D}$ is larger than the width of the occupied conduction band in the leads $\Omega_{L / R}$ [the case of Fig. 2(b)] electron states from $\mu_{L}+\Delta k_{B} T$ to $V_{S D}$ are not occupied. 
The electron tunneling probability through $\Omega$ is calculated using the Green's functions as

$$
T(\omega)=\Gamma_{L}\left(x_{L}, x_{L}^{\prime} ; \omega\right) G^{r}\left(x_{L}^{\prime}, x_{R} ; \omega\right) \Gamma_{R}\left(x_{R}, x_{R}^{\prime} ; \omega\right) G^{a}\left(x_{R}^{\prime}, x_{L} ; \omega\right),
$$

and the current is obtained by integrating over $\omega$, i.e.,

$$
I=\frac{1}{\pi} \int_{-\infty}^{\infty} T(\omega)\left[F_{R}(\omega)-F_{L}(\omega)\right] d \omega .
$$

This whole integral has to be calculated on the real axis, which is not a problem because the integral is evaluated only once after the self-consistent solution of the electron density is reached.

\section{B. Finite-element implementation of the GF formalism}

We have implemented the GF formalism using the FEM. For the FEM implementation we need to write the equations to be solved in the variational form. We start from Eq. (6) and multiply both sides by a continuous well-behaving function $v(x)$ and integrate over $\Omega$. After modifications the equation obtains the form

$$
\begin{aligned}
\int_{\Omega}\{ & -\nabla v(x) \frac{1}{2} \nabla G^{r}\left(x, x^{\prime} ; \omega\right) \\
& \left.+v(x)\left[\omega-V_{e f f}(x)\right] G^{r}\left(x, x^{\prime} ; \omega\right)\right\} d x \\
& +v\left(x_{L}\right) \hat{\Sigma}_{L}^{r}\left(x_{L}, x_{L} ; \omega\right) G^{r}\left(x_{L}, x_{L} ; \omega\right) \\
& +v\left(x_{R}\right) \hat{\Sigma}_{R}^{r}\left(x_{R}, x_{R} ; \omega\right) G^{r}\left(x_{R}, x_{R} ; \omega\right)=v\left(x^{\prime}\right) .
\end{aligned}
$$

Here the self-energy operators $\hat{\Sigma}_{R / L}^{r}$ have the analytic solutions $^{14}$

$$
\hat{\Sigma}_{L / R}^{r}\left(x, x^{\prime} ; \omega\right)=\frac{1}{4} \frac{\partial^{2} g_{L / R}^{e}\left(x, x^{\prime} ; \omega\right)}{\partial x \partial x^{\prime}},
$$

where $g_{L / R}^{e}$ are the retarded Green's functions in the isolated leads $\Omega_{L / R}$ so that they vanish at the boundaries $\partial \Omega_{L / R}$. In our model the potential is constant in the leads so that

$$
g_{L / R}^{e}\left(x, x^{\prime} ; \omega\right)=-\frac{i}{\sqrt{2 \omega}}\left(e^{i \sqrt{2 \omega}\left(x-x^{\prime}\right)}-e^{i \sqrt{2 \omega}\left(x+x^{\prime}-2 x_{L / R}\right)}\right) .
$$

In the FEM the retarded Green's function is expanded in the basis $\phi_{i}$,

$$
G^{r}\left(x, x^{\prime} ; \omega\right) \approx \sum_{i, j=1}^{N} g_{i j}(\omega) \phi_{j}(x) \phi_{i}\left(x^{\prime}\right)
$$

This is an approximation due to the finite number $N$ of the basis functions. Equation (24) is inserted into Eq. (21). The values of the coefficients $g_{i j}$ are then calculated by choosing $v(x)=\phi_{k}(x)$.

We use a basis consisting of the linear functions $\phi^{0}$ and $\phi^{1}$ and of high-order polynomials $\phi^{j}{ }^{22}$ That is,

$$
\phi^{0}(\chi)=\frac{1}{2}(1-\chi), \quad \phi^{1}(\chi)=\frac{1}{2}(1+\chi),
$$

$$
\phi^{j}(\chi)=\sqrt{\frac{1}{2(2 j-1)}}\left[P_{j}(\chi)-P_{j-2}(\chi)\right], \quad j=2,3, \ldots,
$$

where $P_{j}(\chi)$ 's are the Legendre functions of the order $j$. $\phi^{j}(\chi)$ 's are given in the reference element with $\chi=[-1,1]$. The linear functions span the region of two elements, whereas the $j>1$ functions are localized within one element only. The inclusion of the high-order basis functions reduces the number of basis functions needed in order to obtain accurate results. This has a remarkable effect in GF electron structure calculations even for one-dimensional systems. The calculation of the $G^{r}\left(x, x^{\prime} ; \omega\right)$ requires the inversion of a matrix of the size of $N \times N$. The derivatives of the functions with $j>1$ are orthogonal to each other. This makes their use numerically stable and we have implemented elements up to the fifth order.

\section{Wigner function formalism}

We have also implemented the WF formalism for the electron density and the current calculations. Reviews of the WF formalism for studies of open systems can be found in Refs. 7 and 8. There are similarities between our GF and WF implementations in the treatment of the one-dimensional equations resulting from the translational invariance in the directions perpendicular to the electron current. They show up, for example, in the boundary conditions as will be discussed below.

The WF $f(q, p)$ is defined as the Fourier transform of the density matrix $\rho\left(x, x^{\prime}\right)$, i.e.,

$$
f(p, q)=\int_{-\infty}^{\infty} e^{-i p r} \rho\left(q+\frac{1}{2} r, q-\frac{1}{2} r\right) d r
$$

where $q=\frac{1}{2}\left(x+x^{\prime}\right)$ and $r=\left(x-x^{\prime}\right)$ are the new coordinates and the well known phase-space representation $f(q, p)$ is obtained.

In order to use the WF formalism in transport theory we must study the time evolution of the WF. It is calculated from the quantum-mechanical Liouville-von Neumann equation

$$
i \frac{\partial \rho}{\partial t}=[\hat{H}, \rho] \equiv \mathcal{L} \rho
$$

where $\hat{H}$ is the Hamiltonian of the system and $\mathcal{L}$ is the Liouville superoperator. In the WF this equation has the form

$$
\frac{\partial f(q, p)}{\partial t}=-p \frac{\partial f(q, p)}{\partial q}-\int_{-\infty}^{\infty} \frac{1}{2 \pi} V\left(q, p-p^{\prime}\right) f\left(q, p^{\prime}\right) d p^{\prime},
$$

where 


$$
V(q, p)=2 \int_{0}^{\infty} \sin (p r)\left[V_{\mathrm{eff}}\left(q+\frac{1}{2} r\right)-V_{\mathrm{eff}}\left(q-\frac{1}{2} r\right)\right] d r
$$

is a one-dimensional potential kernel. The effect of $V_{\text {eff }}$ is nonlocal, incorporating quantum interference effects. Namely, the potential kernel $V\left(q, p-p^{\prime}\right)$ spreads according to Eq. (28) the $\operatorname{WF} f(q, p)$ among different values of $p$ and adds the interference between alternative paths to the formulation.

The electron density $n_{-}(q)$ and current density $J(q)$ are calculated as

$$
J(q)=\frac{1}{2 \pi} \int_{-\infty}^{\infty} f(q, p) p d p
$$

and

$$
n_{-}(q)=\frac{1}{2 \pi} \int_{-\infty}^{\infty} f(q, p) d p
$$

In an open system we need to make a difference between the incoming and outgoing particles to account for the irreversibility. Our system is one-dimensional, so that $0 \leqslant q \leqslant W_{\Omega}$, and there are two boundaries at $q=0$ and $q=W_{\Omega}$. Here, $W_{\Omega}$ $=x_{R}-x_{L}$ is the width of the central region $\Omega$. Since the characteristics are first-order equations only one boundary value is needed. Moreover, since particles which have $p>0$ are moving in the positive direction on the $q$ axis, we must supply the boundary conditions on the left-hand side boundary $\partial \Omega_{L}$ from where they are originating with the momentum distribution in the reservoir. Similarly particles with $p<0$ are moving in the negative direction on the $q$ axis so that the boundary conditions must be specified on the right-hand side boundary $\partial \Omega_{R}$. This scheme invokes the boundary conditions

$$
\begin{gathered}
f(0, p)_{p>0}=F_{L}\left(\frac{1}{2} p^{2}\right), \\
f\left(W_{\Omega}, p\right)_{p<0}=F_{R}\left(\frac{1}{2} p^{2}\right),
\end{gathered}
$$

where $F_{L / R}$ are as defined in Eq. (11) and take again the integration over the perpendicular energy components into account.

We solve the Wigner transport equation for the steady state with $\partial f / \partial t=0$ numerically using the discretization scheme explained in Ref. 7. The position coordinate $q$ is simply discretized as $N_{q}$ equally spaced points with the spacing $\Delta_{q}=W_{\Omega} /\left(N_{q}-1\right)$. Once $\Delta_{q}$ is fixed the Fourier completeness relation gives the grid spacing $\Delta_{p}$ as a function of $\Delta_{q}$ as

$$
p_{k}=\frac{\pi}{\Delta_{q}}\left(\frac{k-1}{N_{p}}-\frac{1}{2}\right), \quad k=1,2, \ldots, N_{p} .
$$

The derivative of $f(q, p)$ with respect to $q$ in the transport equation (28) has been calculated using the second-order differencing scheme (SDS). That is

$$
\frac{\partial f(q, p)}{\partial q}= \pm\left[\frac{3 f(q, p)-4 f\left(q \pm \Delta_{q}, p\right)+f\left(q \pm 2 \Delta_{q}, p\right)}{2 \Delta_{q}}\right],
$$

where the different signs are chosen according to the signs of $p_{k}$ in such a way that the proper boundary conditions given by Eq. (32) are coupled to the transport equation. It has been shown by Buot and Jensen ${ }^{24,25}$ that the SDS gives considerably better results than the conventional upwind-downwind differencing scheme (UDS).

\section{Differences between the Green's function and Wigner function formalisms}

The GF and WF formalisms are related. Namely, we can first calculate the density operator in the GF formalism as

$$
\rho\left(x, x^{\prime}\right)=-i \int_{-\infty}^{\infty} G^{<}\left(x, x^{\prime} ; \omega\right) d \omega .
$$

Then the coordinate transformation is performed and we get the $\mathrm{WF} f(p, q)$. If $f(p, q)$ is calculated in this way Eqs. (30) and (31) of the WF formalism give exactly the same results as Eqs. (9) and (20) of the GF formalism.

The differences in the results of the two formalisms are caused by the calculation of $f(p, q)$ in the WF formalism by using the Liouville-von Neumann equation. For an infinite system the results would still be equal but a finite calculation region causes the differences. Namely, the WF formalism does not have similar totally open boundary conditions as the GF formalism. Differences arise in this case also because there is no energy dependence in the Liouville-von Neumann equation whereas $G^{<}$depends on $\omega$.

Using the GF formalism typically means that we have to calculate a lot of information which is of no further use. The calculation of $G^{R}\left(x, x^{\prime} ; \omega\right)$ using $N$ basis functions means inverting a $N \times N$ matrix. This requires the solution of $N$ linear equations each having $N$ unknown variables. The coefficient matrix is sparse, including only overlapping terms of the basis functions. In order to calculate the electron density we have to integrate over $\omega$, which results in calculating $G^{r}\left(x, x^{\prime} ; \omega\right)$ many times. Luckily, in the integrals $n_{-2}$ and $n_{-3}$ [Eqs. (17) and (18)] we need only two linear equations for $G^{r}\left(x_{L}, x\right)$ and $G^{r}\left(x_{R}, x\right)$.

In the WF formalism we need to solve for the Wigner function, which depends on two variables $p$ and $q$. Because of the use of the discretization form of Eq. (34) the dependence between these variables is more complicated than that between $x$ and $x^{\prime}$ in the GF formalism. This means that we solve a set of linear equations which includes $N_{p} \times N_{q}$ unknown variables. The coefficient matrix has a belt type filling where the width of the belt is large. This makes the linear equation hard to solve.

\section{RESULTS}

In this section we give results of our electronic structure and electron current calculations for the magnetic RTD structure described in Sec. II A. We concentrate on the feasibility 


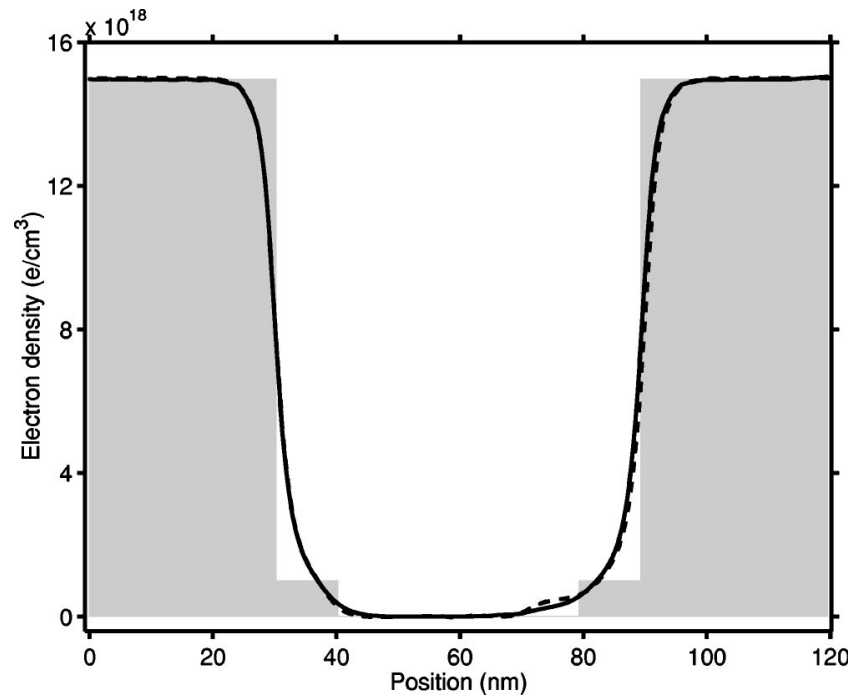

FIG. 3. Electron density calculated using the GF (solid line) and the WF (broken line) formalisms. The values of the temperature, magnetic field, and bias voltage used are $4.2 \mathrm{~K}, 6 \mathrm{~T}$, and $0.15 \mathrm{~V}$, respectively. The gray areas denote level of positive back ground charge.

of the GF and WF formalisms to model this kind of systems. In the first subsection this is done by comparing the results of the two formalisms with each other. In the second subsection we compare our results with those measured by Slobodskyy et $a .^{6}$ and thereby analyze the electronic structure of the actual device. Finally, we make predictions for the spinpolarized current when structure parameters of the magnetic RTD are varied.

\section{A. Comparison between the Green's function and Wigner function formalisms}

The electron density and the effective potential corresponding to the $0.15 \mathrm{~V}$ bias voltage and calculated using the GF and the WF formalisms are shown in Figs. 3 and 4, respectively. The two formalisms give very similar results. There are Friedel oscillations in the electron density in the leads but their amplitude is so small that they are not visible on the scale used. The electron density drops close to zero in the undoped regions. For this value of $V_{S D}$ some of the lowenergy resonance states are occupied. However, these states cause only a small density increment between the potential barriers. The electron density shows in both formalisms a small asymmetric behavior which becomes clearer with increasing $V_{S D}$.

The effective potential in Fig. 4 rises strongly at the interfaces between the doped and undoped materials. Within the undoped region the potential changes then rather linearly due to the applied bias voltage. The spin splitting of the potential due to the external term of Eq. (1) does not propagate out of the quantum well region. Our GF and WF calculations do not include inelastic scattering. That would be important for finding self-consistent solutions if, on the higher potential side of the barriers, there are "notch" states below the band of occupied electron states. ${ }^{7}$ In our case

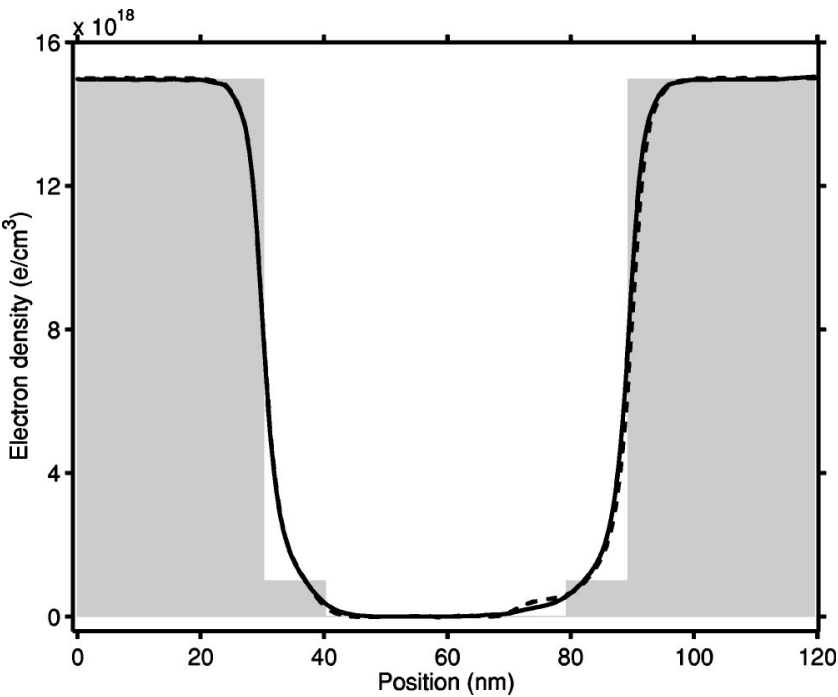

FIG. 4. Effective potential calculated using the GF (solid line) and the WF (dashed line) formalisms. The values of the temperature, magnetic field, and bias voltage used are $4.2 \mathrm{~K}, 6 \mathrm{~T}$, and 0.15 $\mathrm{V}$, respectively. The effective potentials for spin-up and spin-down electrons differ remarkably only between the potential barriers.

"notch" states do not appear because of the strong potential rise due to the undoped material layers.

The current through our magnetic RTD structure is shown in Fig. 5 as function of the bias voltage $V_{S D}$. The spin-up and spin-down contributions, split strongly by the magnetic field of $6 \mathrm{~T}$, show the typical RTD behavior. The most prominent peaks at around $0.14-0.18 \mathrm{~V}$ actually correspond to the second lowest resonance states in energy. Most of the conduction takes place close to the right Fermi level $\mu_{R}$ because the large undoped region diminishes strongly the tunneling probability at lower energies. For this reason also the current

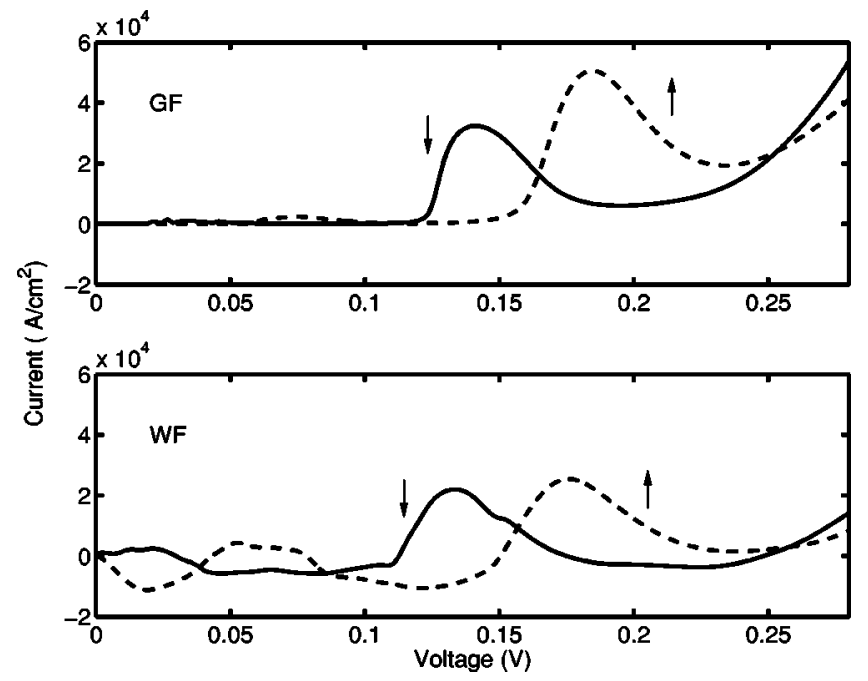

FIG. 5. Current through the magnetic RTD structure as a function of the bias voltage. The contributions due to spin-down (solid line) and spin-up (dashed line) electrons are shown. The results in the upper and lower panels are calculated using the GF and the WF formalisms, respectively. The values of the temperature and magnetic field used are $4.2 \mathrm{~K}$ and $6 \mathrm{~T}$, respectively. 


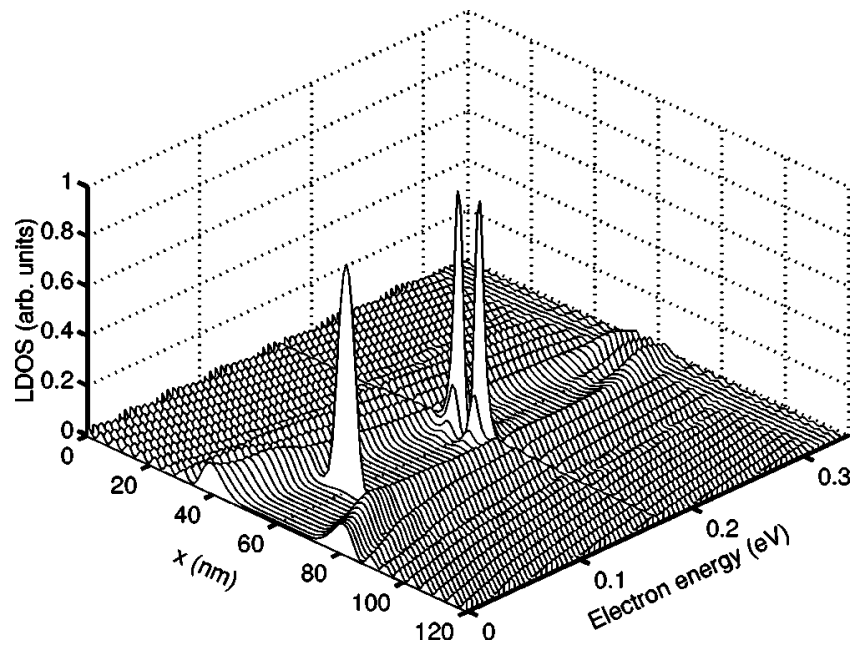

FIG. 6. LDOS of the GF formalism as a function of the position $x$ along direction of the current and the energy $\omega$. The values of the temperature and magnetic field are $4.2 \mathrm{~K}$ and $0 \mathrm{~T}$, respectively.

peaks due to the lowest-energy resonances at around 0.03$0.07 \mathrm{~V}$ are hardly visible in the GF results. They are clearer in the WF results, but this is partly due to numerical difficulties as will explain below. In the WF formalism the current may even change its direction and have negative values as seen in the lower panel of Fig. 5.

The two resonance states per spin in the voltage region of $0-0.25 \mathrm{~V}$ can be clearly seen in the local density of states (LDOS) calculated in the GF formalism. Figure 6 shows the LDOS for the zero-magnetic field and the zero-bias voltage case. In the absence of the magnetic field, there is no difference between the spin-up and spin-down electron states. The first and the second resonance correspond to the quantum well states with zero and one node plane parallel to the layer structure, respectively. We can also see that especially at low energies the LDOS enhances just outside the undoped region.

The GF and the WF formalisms give almost equal electron densities and effective potentials as demonstrated in Figs. 3 and 4. However, the requirements to reach similar numerical convergences are very different. In the GF calculations we have used 36 fifth-order elements corresponding to 181 basis functions. A further increase of the number of elements does not change the results. In the WF calculations we have used as many as $N_{q}=320$ and $N_{p}=300$ discretization points. This means that the number of unknown variables in the linear equations to be solved is of the order of $10^{5}$. If we reduce the number of discretization points from this magnitude, the effective potential in the RTD region moves down becoming saggy. The very different numerical characteristics of our WF and the GF calculations demonstrate the efficiency of the high-order polynomial basis used in our GF implementation.

In the current-voltage curves of Fig. 5 the differences between the WF and GF formalisms are more visible than in the electron density of Fig. 3 although the formalisms give the same qualitative behavior. The resonance peaks of the WF results are located at slightly lower bias voltages (energies) than those in the GF results. This is in accordance with the lower effective potential in the WF calculations (see Fig. 4). The GF formalism always gives by definition a positive current whereas the current calculated by using the WF formalism may become negative. This is a well-known artifact of the WF formalism. If we reduce the number of discretation points the current attains even more negative values. This implies that the WF results of Fig. 5 are not numerically fully convergent. However, with the present computer memory limits we cannot increase the number of discretization points much beyond $10^{5}$. At this stage the WF calculation begins to require also more CPU time than the GF calculations. This is somewhat surprising because one would think that the less approximative GF formalism would be computationally heavier. The use of a more accurate differencing scheme could improve the WF results without increasing the number of the discretization points, but this would increase the filling of the coefficient matrix to be inverted and the CPU time needed.

The numerical problems in the WF calculations are caused by the narrow resonance peaks. A Fourier transformation has to be evaluated over electron energies, and the resonance peaks require a remarkable increase of discretation points. Indeed, Fig. 5 shows that the current from the WF calculations for both spins oscillates strongly in the region of the first resonance peak, which is very narrow. The problem is not faced in the electron density calculations, because the contributions of the resonance states to the total electron density are small. Our real-space GF implementation can handle the narrow resonance peaks better because we use the adaptive Simpson integral routine to calculate the density integrals $n_{-2}, n_{-3}$, and the current integral along the real $\omega$ axis and because the integral $n_{-1}$ is performed in the complex plane where the resonance peaks are broadened.

\section{B. Comparison to the experiments}

We compare our calculated results with the recent measurements by Slobodskyy et al. ${ }^{6}$ In particular, we have calculated the current vs bias voltage curves using the same magnetic field and temperature values as they have used.

The results of the WF and GF calculations for the current as a function of the bias voltage are shown in Fig. 7 corresponding to different magnetic fields and in Fig. 8 to different temperatures. As was mentioned above we have chosen the height of the potential barriers so that the widths of the two prominent spin-up and spin-down peaks from our GF calculations agree at low temperatures and high magnetic fields with experiment as well as possible. With this fitting only, our predictions for the magnetic field and temperature dependences are rather similar to experiments. The spin-up and spin-down contributions separate in similar ways with increasing magnetic field and merge together with an increasing temperature. These overall features are related to the dependence of the spin splitting $\Delta E$ on magnetic field and temperature in Eq. (1). One should note that the distances between the spin-up and spin-down peaks in the current are by a factor of 2 larger than $\Delta E$ (see the values in Table II). The reason is that part of the voltage is dropped over the first potential barrier. This fact was taken into ac- 

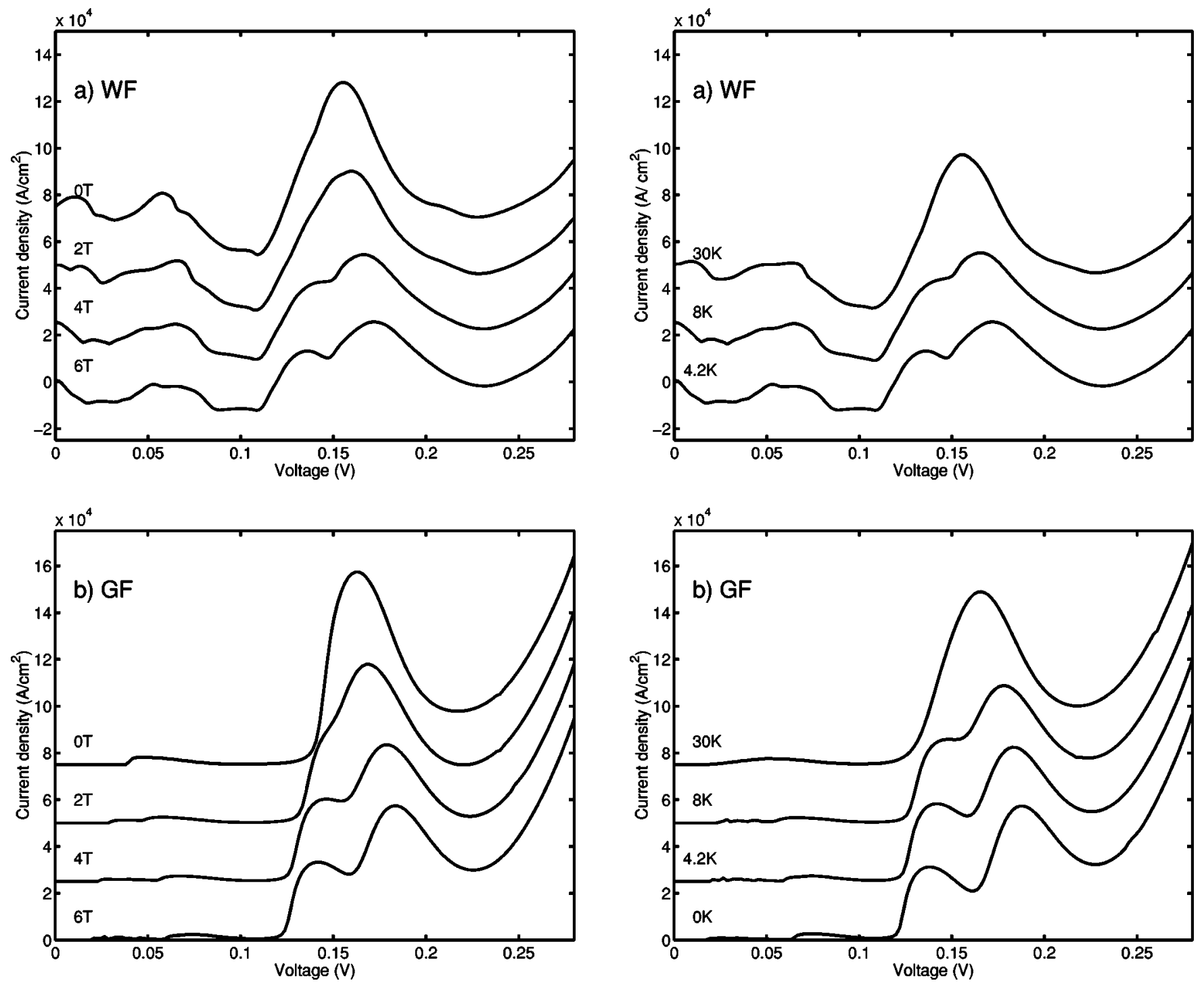

FIG. 7. Current through the magnetic RTD structure as a function of the bias voltage and different values of the magnetic field. The structure is defined in Fig. 1 and in Table I. The results in the panels (a) and (b) are calculated using the WF and the GF formalisms, respectively. The temperature is $4.2 \mathrm{~K}$. For clarity, the successive curves are shifted by $2.5 \times 10^{4} \mathrm{~A} / \mathrm{cm}^{2}$ with respect to each other.

count by Slobodskyy et $a .^{6}$ by a lever arm of 2.1 when comparing the measured voltage splitting with the theoretical $\Delta E$. Our simulations confirm the magnitude of the lever arm.

Even if the shapes of the calculated current-voltage curves are similar to those in the experiment, the calculated current vales are up to five orders in magnitude too large. (We assume that the area of the experimental device is $100 \mu \mathrm{m}$ $\times 100 \mu \mathrm{m}$.) The current amplitudes predicted by our WF and GF calculations agree reasonably well and they are similar in magnitude to currents calculated for typical RTD structures in Refs. 7 and 8. This indicates that the reason for the theoryexperiment disagreement is in fundamental approximations. It is well known that the density-functional theory gives about two orders of magnitude too large tunneling currents through molecules in comparison with experiments. ${ }^{26} \mathrm{We}$

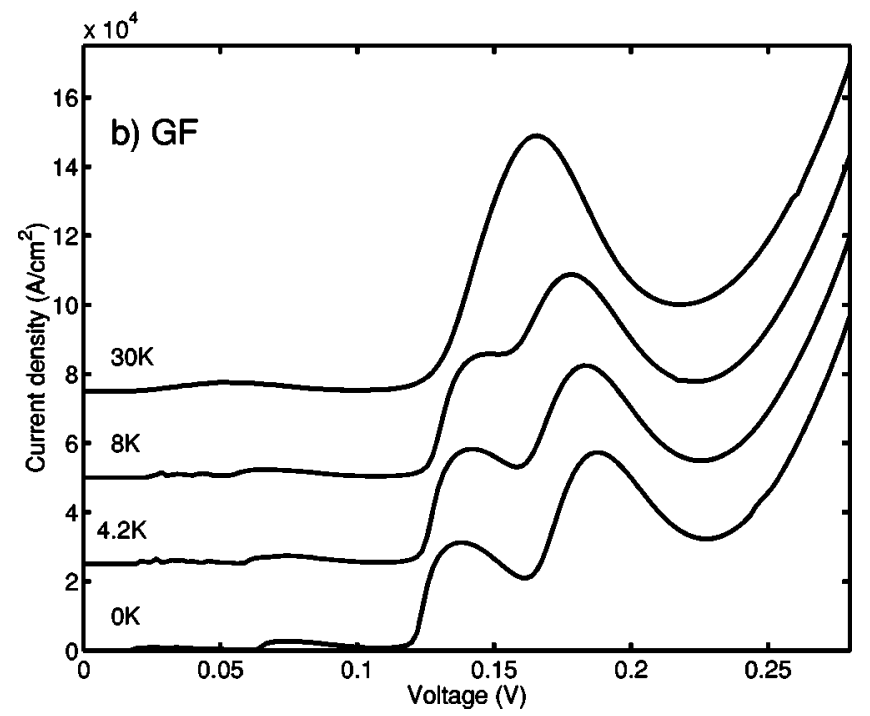

FIG. 8. Current through the magnetic RTD structure as a function of the bias voltage in different temperatures. The structure is defined in Fig. 1 and in Table I. The results in the panels (a) and (b) are calculated using the WF and the GF formalisms, respectively. The magnetic field is $6 \mathrm{~T}$. For clarity, the successive curves are shifted by $2.5 \times 10^{4} \mathrm{~A} / \mathrm{cm}^{2}$ with respect to each other.

have also used the effective mass approximation which is also expected to affect the magnitude of the current.

However, the present large discrepancy calls for investigation of other assumptions we have done. One reason could be that the high doping in the leads results in the impurity band formation which we have not taken into account, but used the parabolic free energy band with the effective mass of the conduction band. If the improved description of the bands moved the first resonance peak to be the relevant one in the correct bias voltage region, the current magnitude might also decrease. We cannot easily use different effective masses in the different regions of the computations. Therefore we mimic the effect of the formation of the impurity band by lowering the external potential in the leads with respect to that in the undoped regions. However, even a lowering of the order of the bandwidth in the leads does not 


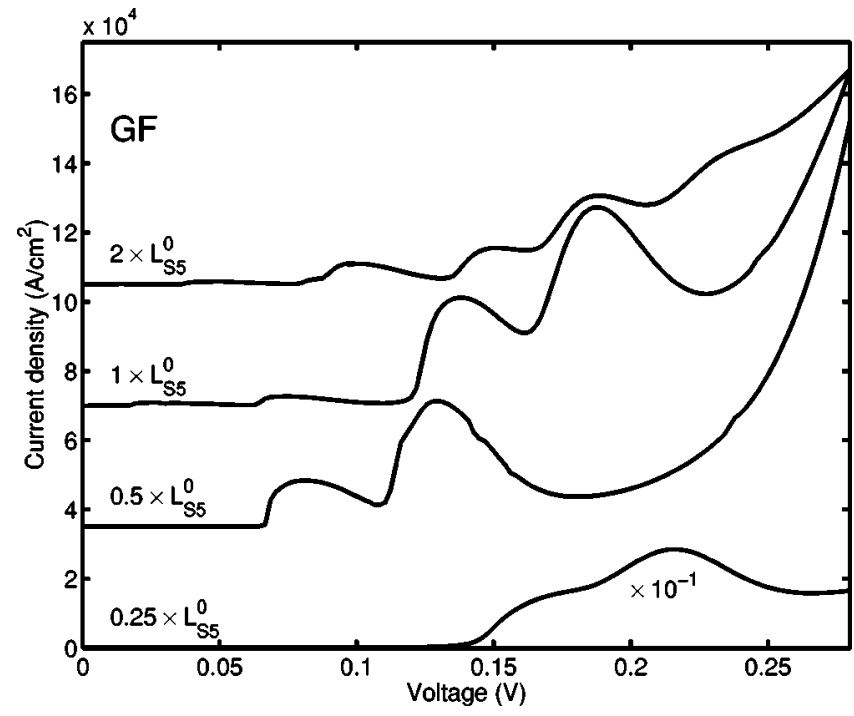

FIG. 9. Current through a magnetic RTD structure as a function of the bias voltage. The structure is defined in Fig. 1 and in Table I with the exception that the width of the quantum well (region S5) is scaled relative to the original width $L_{S 5}^{0}$. The results are calculated using the GF formalism and they correspond to the the magnetic field of $6 \mathrm{~T}$ and zero temperature. For clarity, the successive curves are shifted by $3.5 \times 10^{4} \mathrm{~A} / \mathrm{cm}^{2}$. The lowest curve for $0.25 \times L_{S 5}^{0}$ is scaled by a factor of $1 / 10$.

result in an appreciable shift of the total effective potential between the lead and the central region. This is because in our self-consistent calculations the ensuing charge transfer causes a change in the electrostatic potential opposing the shift of the external potential. Thus, the positions of the resonance peaks on the bias voltage axis are mainly derived from the width of the quantum well and from the fact that in equilibrium the first resonance state has to be mostly unoccupied, i.e., slightly above the Fermi level.

The remaining difference of about $30 \mathrm{mV}$ between the positions of the theoretical current peaks (Figs. 7 and 8) and those of the measured ones, so that the theoretical peaks are at higher voltages, may be due to several effects. First of all, uncertainty in the thickness of the quantum well affects strongly the resonance positions. The value of the series contact resistance in the measurements, the lack of inelastic scattering, and the use of a constant effective electron mass in our modeling may also shift the peaks. However, the energy shifts due to these uncertainties are expected to be smaller than the distances between the resonances for each spin direction: the distance between the first and the second resonance is about $100 \mathrm{mV}$ (see Fig. 6). Therefore our conclusion is that the current peaks seen in the measurements by Slobodskyy et al. correspond to the second resonance state in energy. The fact that there are no traces of the first resonances in the experiment is in agreement with the small intensity of the first peaks in comparison with that of the second peaks in our GF calculations.

\section{Effects of the width of the potential well}

In the previous sections we have concentrated on modeling and analyzing the magnetic RTD structure by Slobod-

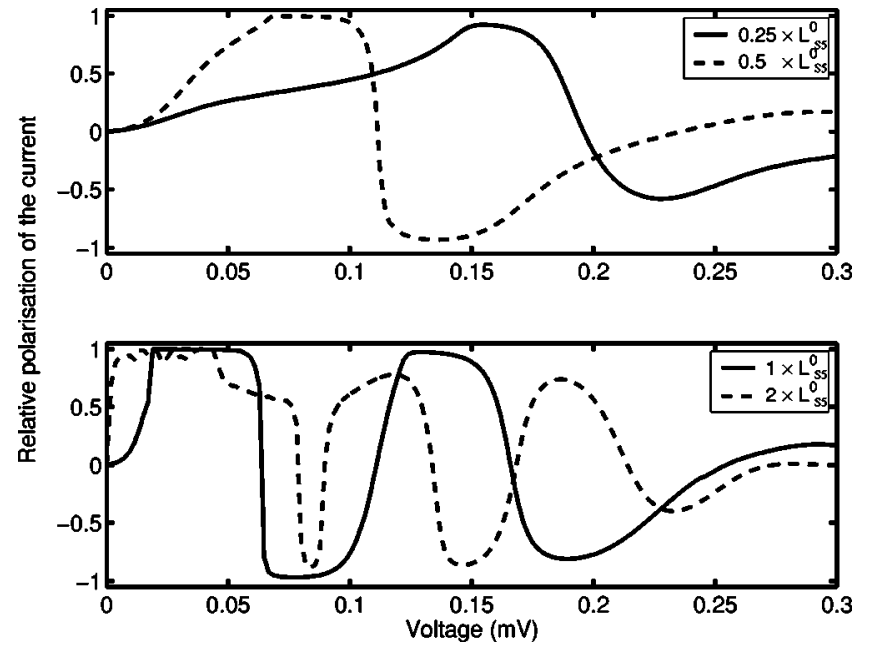

FIG. 10. Relative polarization of the current through a magnetic RTD structure as function of the bias voltage. The curves correspond to the total currents shown in Fig. 9. See also the caption of Fig. 9.

skyy et al. Next we predict how the geometry of the device affects the spin polarization of the current. The obvious parameter to be varied is the width of the quantum well because it determines the positions of the resonances on the energy axis and affects strongly even the qualitative features of the current-voltage curves. Thus, in the following we keep the width and height of the potential barriers the same as in the previous calculations and vary the width $L_{S 5}$ of the potential well using the values $L_{S 5}=0.25 L_{S 5}^{0}, 0.5 L_{S 5}^{0}$, and $2 L_{S 5}^{0}$, where $L_{S 5}^{0}$ is the original width of $9 \mathrm{~nm}$ (Table I).

The current as a function of the bias voltage for the different widths of the potential well is shown in Fig. 9. We see that the narrowing of the well moves resonance states towards higher voltages and the first resonance peak for each spin becomes active, i.e., its contribution to the total current becomes evident. We also note that the magnitude of the current increases rapidly with decreasing potential well width. The increase of the potential well width to $2 L_{S 5}$ causes the resonances to come closer to each other and overlap more strongly. This might be an undesirable feature for device applications.

Another view of the functioning of the magnetic RTD structure as a spin switch is given in Fig. 10 showing the relative polarization of the current, i.e., the difference between the spin-up and spin-down electron currents divided by the total current. For structures with very narrow potential wells the polarization is reduced, although the magnitude of the current is large (Fig. 9). For structures with wide potential wells the spin splitting for a given magnetic field strength may become comparable with the distances between the resonances. This causes a complicated polarization structure as a function of the bias voltage as can be seen in Fig. 10 for the widest potential well of $2 L_{S 5}$. In conclusion, for a given magnetic field strength there is an optimum potential well width with good polarization properties and high current intensity. In the structures studied above and in the strong field of $6 \mathrm{~T}$ it would be approximately $0.3-0.5$ times the original width $L_{S 5}^{0}$. 


\section{CONCLUSIONS}

We have used the semiclassical Wigner function formalism and the quantum-mechanical Green's function formalism to model spin-dependent electron transport through a magnetic resonant-tunneling diode structure. Our calculations are based on the self-consistent solution of the electronic structures and currents within the density-functional theory. Both formalisms give very similar results for the electron density, but the current is more sensitive to the formalism used. We have traced the differences to be largely due to the numerical accuracy which is costly to achieve in the conventional implementation of the Wigner function formalism. Surprisingly, the Green's function formalism implemented by using the finite-element method requires less computer resources and numerically converged results are obtained.

Using the two schemes, especially the Green's function formalism, we have analyzed the recent measurements by Slobodskyy et al. ${ }^{6}$ for an actual magnetic resonant-tunneling diode. The magnetic-field and temperature dependencies in the measured current-bias-voltage curves are well reproduced. The two-peak structure is found to result from the spin-split resonance second lowest in energy. We show that for a given strength of the magnetic field causing the spin splitting there exists an optimum width for the quantum well so that high spin polarization and current intensity are achieved.

\section{ACKNOWLEDGMENTS}

We acknowledge the generous computer resources from the Center for Scientific Computing, Espoo, Finland. This research has been supported by the Academy of Finland through its Centers of Excellence Program (2000-2005), by the Finnish Cultural Foundation and by the Vilho, Yrjö, and Kalle Väisälä Foundation. We are grateful to Per Hyldegaard, Pekka Kuivalainen and Ville Havu for useful discussions.
${ }^{1}$ H. J. Zhu, M. Ramsteiner, H. Kostial, M. Wassermeier, H. P. Schonherr, and K. H. Ploog, Phys. Rev. Lett. 87, 016601 (2001).

${ }^{2}$ V. F. Motsnyi, J. De Boeck, J. Das, W. Van Roy, G. Borghs, E. Goovaerts, and V. I. Safarov, Appl. Phys. Lett. 81, 265 (2002).

${ }^{3}$ R. Fiederling, M. Keim, G. Reuscher, W. Ossau, G. Schmidt, A. Waag, and L. W. Molenkamp, Nature (London) 402, 787 (1999).

${ }^{4}$ Y. Ohno, D. K. Young, B. Beschoten, F. Matsukura, H. Ohno, and D. D. Awschalom, Nature (London) 402, 790 (1999).

${ }^{5}$ G. Schmidt, G. Richter, P. Grabs, C. Gould, D. Ferrand, and L. W. Molenkamp, Phys. Rev. Lett. 87, 227203 (2001).

${ }^{6}$ A. Slobodskyy, C. Gould, T. Slobodskyy, C. R. Becker, G. Schmidt, and L. W. Molenkamp, Phys. Rev. Lett. 90, 246601 (2003).

${ }^{7}$ W. R. Frensley, Rev. Mod. Phys. 62, 745 (1990).

${ }^{8}$ C. Jacoboni and P. Bordone, Rep. Prog. Phys. 67, 1033 (2004).

${ }^{9}$ S. Datta, Electronic Transport in Mesoscopic Systems (Cambridge University Press, Cambridge, 1995).

${ }^{10}$ Roger Lake, Gerhard Klimeck, R. Chris Bowen, and Dejan Jovanovic, J. Appl. Phys. 817845 (1997).

${ }^{11}$ K. M. Indlekofer, J. Lange, A. Förster, and H. Lüth, Phys. Rev. B 53, 7392 (1996).

${ }^{12}$ Gianluca Stefanucci and Carl-Olof Almbladh, Phys. Rev. B 69, 195318 (2004).

${ }^{13}$ M. B. Unlu, B. Rosena, H.-L. Cuia, and P. Zhaob, Phys. Lett. A
327, 230 (2004).

${ }^{14}$ P. Havu, V. Havu, M. J. Puska, and R. M. Nieminen, Phys. Rev. B 69, 115325 (2004).

${ }^{15}$ P. Havu, M. J. Puska, R. M. Nieminen, and V. Havu, Phys. Rev. B 70, 233308 (2004).

${ }^{16}$ S. Lee, F. Michl, U Rössler, M. Dobrowolska, and J. K. Furdyna, Phys. Rev. B 57, 9695 (1998).

${ }^{17}$ Landolt-Börnstein, Intrinsic Properties of Group IV Elements and III-V, II-VI, and I-VII Compounds, edited by O. Madeling, New Series Group III, Vol 22, Pt. a (Springer, Berlin, 1987).

${ }^{18}$ M. Kim, C. S. Kim, S. Lee, J. K. Furdyna, and M. Dodrowolska, J. Cryst. Growth 214/215, 325 (2000).

${ }^{19}$ C. Chauvet, E. Tournie, and J.-P. Faurie, Phys. Rev. B 61, 5332 (2000).

${ }^{20}$ D. M. Ceperley and B. J. Alder, Phys. Rev. Lett. 45, 566 (1980); J. P. Perdew and A. Zunger, Phys. Rev. B 23, 5048 (1981).

${ }^{21}$ J. Arponen, P. Hautojärvi, R. Nieminen, and E. Pajanne, J. Phys. F: Met. Phys. 3, 2092 (1973).

${ }^{22} \mathrm{C}$. Schwab, $p$ - and hp-Finite Element Methods-Theory and Applications in Solid and Fluid Mechanics (Clarendon, Oxford, 1998).

${ }^{23}$ J. C. Egues, Phys. Rev. Lett. 80, 4578 (2000).

${ }^{24}$ K. L. Jensen and F. A. Buot, J. Appl. Phys. 67, 2153 (1990).

${ }^{25}$ F. A. Buot and K. L. Jensen, Phys. Rev. B 42, 9429 (1990).

${ }^{26}$ See, for example, M. Di Ventra, S. T. Pantelides, and N. D. Lang, Phys. Rev. Lett. 84, 979 (2000). 